EXTENDED REPORT

\title{
Serum prorenin levels and diabetic retinopathy in type 2 diabetes: new method to measure serum level of prorenin using antibody activating direct kinetic assay
}

\author{
H Yokota, F Mori, K Kai, T Nagaoka, N Izumi, A Takahashi, T Hikichi, A Yoshida, F Suzuki, \\ Y Ishida
}

Br J Ophthalmol 2005;89:871-873. doi: 10.1136/bjo.2004.056580

See end of article for authors' affiliations

Correspondence to: Harumasa Yokota, MD Department of Ophthalmology Asahikawa Medical College, 2-1-1-1 Midorigaoka Higashi, Asahikawa, 078-8510 Japan; atokoy18@ asahikawa-med.ac.jp

Accepted for publication 1 December 2004
Aim: To investigate the serum levels of prorenin and its correlation with the severity of diabetic retinopathy (DR).

Methods: 248 patients with diabetes and 108 control subjects were divided into four groups: no-DR $(n=146)$, no proliferative diabetic retinopathy (no-PDR) $(n=78)$, PDR $(n=24)$, and controls $(n=108)$. Serum levels of prorenin from all subjects were measured using the new antibody activating direct kinetic (AAD-PR) assay. The serum prorenin levels were compared among the groups.

Results: The serum levels of prorenin in the control, no-DR, no-PDR, and PDR groups, respectively, were 109.1 (66.1), 194.6 (160.4), 271.5 (220.3), and 428.4 (358.4) pg/ml (mean (SD)). Prorenin in the PDR group was remarkably high compared with the control and no-DR groups $(p<0.0001)$ and with the noPDR group $(p=0.002)$. Serum levels of prorenin increased with increasingly severe retinopathy. No correlation was found between the prorenin level and the duration of disease or $\mathrm{HbA}_{1 \mathrm{c}}$.

Conclusions: The serum levels of prorenin in patients with PDR were found to be markedly high using the AAD-PR assay. Increased levels of prorenin in diabetes may have an important role in the pathogenesis of DR.
D abetic retinopathy (DR) is a major cause of blindness worldwide. Although strict glycaemic control is thought to be essential to prevent the occurrence of DR, ${ }^{1}$ some cases unfortunately develop advanced proliferative diabetic retinopathy $(\mathrm{PDR}){ }^{2}$ In fact, because it is difficult to confirm if diabetic microangiopathy including retinopathy is progressive or not, a useful predictor that is well correlated with the occurrence of diabetic microangiopathy is needed to prevent the development of diabetic microvascular complications.

Prorenin is an inactive precursor of renin. The circulating prorenin level is five to 10 times higher than the active form of renin. Although little is known about the biological function of prorenin, it reportedly increases in diabetes and is associated with the occurrence of DR and nephropathy. ${ }^{3-5}$ Furthermore, in adolescents with diabetes, higher serum levels of prorenin occur several years before diabetic nephropathy ${ }^{6-8}$ and retinopathy. ${ }^{9}$ This modulation of prorenin in diabetes indicates that prorenin is involved in the occurrence and the progression of diabetic microangiopathy. Although measuring prorenin seems to be a good method to determine if diabetic microangiopathy is present or not, the method of measuring prorenin in previous reports has been complicated. Until recently, the level of prorenin was determined by measuring the total renin level and subtracting the active rennin level. ${ }^{10-12}$ Total renin was measured after activating inactive prorenin by trypsin or non-proteolytically. At the same time, active renin was measured independently, and the difference in the levels between total renin and active renin was defined as the prorenin level.

A new method called the antibody activating direct prorenin (AAD-PR) assay, developed by Suzuki et al, ${ }^{13}$ enables direct measurement of the concentration of prorenin using an antibody to the prorenin profragment, which detects prorenin in serum and confirms the complex to the prorenin. This complex has renin-like activity-that is, the ability to convert angiotensinogen to angiotensin I. The generated angiotensin I is measured with the enzyme linked immunosorbent assay. The prorenin level can be calculated by the amount of generated angiotensin I. The AAD-PR assay was reported to have higher sensitivity than previous methods. ${ }^{14}$

In this study, we focused on the relation between the serum levels of prorenin and the severity of DR. We measured serum levels of prorenin in patients with type 2 diabetes and estimated the clinical implication of prorenin in DR using the AAD-PR assay.

\section{PATIENTS AND METHODS}

In all, 248 patients with diabetes and 108 control subjects from Asahikawa Medical College Hospital were included. The control subjects had a normal examination that included urinalysis, blood chemistry, and blood pressure measurement and had never had type 2 diabetes. Patients with diabetes who were followed by physicians at Asahikawa Medical College Hospital all satisfied the criteria for diagnosis of diabetes by the World Health Organization. The subjects received a detailed explanation of the aims of the study and provided informed consent. This study protocol was reviewed by the ethics committee of our institution. All procedures adhered to the tenets of the Declaration of Helsinki.

The subjects were divided into four groups: patients without DR (no-DR group), those with retinopathy but no proliferative DR (no-PDR group), patients with proliferative DR (PDR group), and controls. The characteristics of these groups are shown in table 1. Sera were obtained from all subjects and then treated as described by Kawazu et al to measure the serum levels of prorenin. ${ }^{14}$ The distribution of serum prorenin levels in the four groups was compared using one way of analysis variance and Scheffe's test. A p value of

Abbreviations: $A A D-P R$, antibody activating direct prorenin; $D R$, diabetic retinopathy; PDR, proliferative diabetic retinopathy; RAS, reninangiotensin system 
Table 1 Characteristics of the study groups (SD)

\begin{tabular}{lllll}
\hline & Control & No-DR & No-PDR & PDR \\
\hline Number (male/female) & $108(62 / 46)$ & $146(70 / 76)$ & $78(42 / 36)$ & $24(13 / 11)$ \\
Age (years) & $55.9(15.5)$ & $58(13.3)$ & $60.0(13.3)$ & $56.1(10.1)$ \\
Duration of diabetes (years) & & $8.1(6.9)$ & $13.3(7.2)$ & $14.5(8.4)$ \\
$\mathrm{HbA}_{1 \mathrm{lc}}(\%)$ & & $7.5(1.7)$ & $8.2(1.8)$ & $7.6(1.3)$ \\
Systolic blood pressure (mm Hg) & $124.0(11.5)$ & $123.2(15.3)$ & $131.2(16.0)$ & $134.0(14.2)$ \\
Diastolic blood pressure (mm Hg) & $75.8(3.7)$ & $72.5(4.6)$ & $70.9(9.7)$ & $75.5(9.9)$ \\
\hline
\end{tabular}

0.05 or lower was considered significant. The Pearson correlation coefficient $(r)$ was calculated to determine whether there were close associations among the variables.

\section{RESULTS}

The characteristics of the subjects are shown in table 1 . There is no statistical difference between males and females, which has been reported to affect the serum levels of prorenin. ${ }^{14}$ Systolic blood pressure and diastolic blood pressure were not significantly different among the four groups. The distribution of the serum levels of prorenin in the four groups is shown in figure 1 . The serum levels of prorenin in the control, no-DR, no-PDR, and PDR groups were 109.1 (66.1), 194.6 (160.4), 271.5 (220.3), and 428.4 (358.4) pg/ml (mean (SD)), respectively. The serum levels of prorenin were markedly higher in the PDR group than in the control and no-DR groups $(p<0.0001$, Scheffe's test $)$ and the no-PDR $(p=0.002)$ group. The serum levels of prorenin were higher with increasingly severe retinopathy. No significant correlation was found between the serum prorenin level and disease duration $(r=0.17, \mathrm{p}=0.04)$ or the $\mathrm{HbA}_{\mathrm{lc}}$ level $(r=0.05$, $\mathrm{p}=0.56)$.

\section{DISCUSSION}

In this study, we evaluated the serum levels of prorenin in patients with diabetes using the newly developed AAD-PR assay. This study showed that the serum levels of prorenin in patients with diabetes with PDR were remarkably higher than in the normal healthy subjects, patients without DR, and those with no PDR. The serum concentration of prorenin in patients with diabetes was higher than in control subjects, and a high serum concentration of prorenin in patients with diabetes increased with increasingly severe retinopathy. These results supported previous reports that had shown the clinical implication of prorenin in the occurrence and the development of diabetic microangiopathy. ${ }^{3-9} 15$

Recent studies investigated the relation between the concentration of prorenin and the occurrence or the development

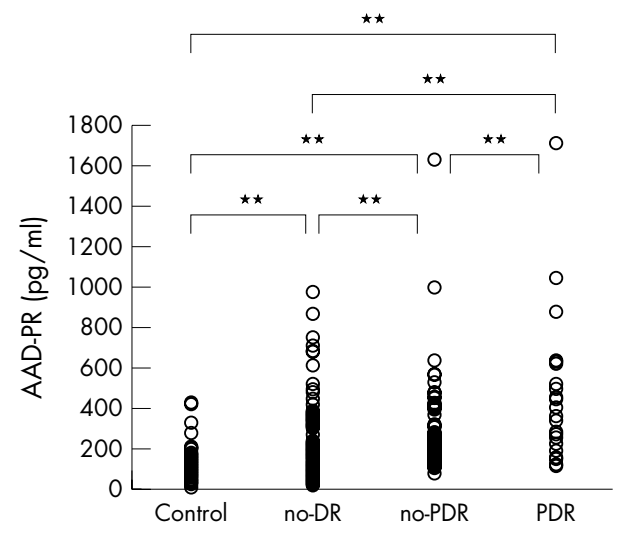

Figure 1 The distribution of serum levels of prorenin; ${ }^{*} p<0.05$, ${ }^{* *} p<0.01$. of DR. ${ }^{3-9}{ }^{15}$ Franken et al reported that a high plasma prorenin level is associated with DR, particularly PDR. ${ }^{4}$ Makimattila et al reported that the serum total renin level increased and was a useful marker of activity and the severity of DR..$^{15}$ Total renin is composed of renin and prorenin, and $90 \%$ of total renin is prorenin. ${ }^{16}$ The active renin level in diabetes does not increase. ${ }^{17}{ }^{18}$ An increase in the total renin level was thought to be the result of the increased level of prorenin in diabetes. These reports showed the close relation between the concentration of prorenin and the severity of $\mathrm{DR}^{4}{ }^{15}$ and supported our results. Although those previous reports showed higher levels of prorenin in diabetes with retinopathy, the conventional measurement method was more complicated and less sensitive for determining the concentration of prorenin than the AADPR assay..$^{14}$

In the present study, we showed that there was no close relation between the serum levels of prorenin and $\mathrm{HbA}_{\mathrm{lc}}$ or duration of diabetes. Franken et al reported that the plasma concentration of prorenin was not correlated with $\mathrm{HbA}_{\mathrm{lc}}$ and the duration of diabetes. ${ }^{5}$ On the other hand, Makimattila et al reported that the serum concentration of total renin was correlated with $\mathrm{HbA}_{\mathrm{Ic}}{ }^{15}$ Luetscher et al also demonstrated a positive correlation between $\mathrm{HbA}_{1 \mathrm{c}}$ and the plasma concentration of prorenin. ${ }^{3} \mathrm{HbA}_{\mathrm{lc}}$ and the duration of diabetes are key risk factors for diabetic microangiopathy and are thought to be associated with the occurrence of DR. ${ }^{19}$ Although $\mathrm{HbA}_{\mathrm{lc}}$ is an important indicator for determining the degree of glycaemic control in diabetes, this is not sufficient to be associated with the occurrence and the severity of DR. ${ }^{20}$ Higher serum levels of prorenin in diabetes might be more appropriate for estimating the occurrence and the severity of DR than $\mathrm{HbA}_{1 \mathrm{c}}$. In this study, the duration of diabetes was longer in patients with PDR than other patients who had no retinopathy or in whom retinopathy was not proliferative; however, there was no close relation between the serum levels of prorenin and the duration of diabetes. Duration, as mentioned previously, is also an important key factor for the occurrence of $\mathrm{DR},{ }^{19}$ but it does not seem to affect the serum concentration of prorenin.

In this study, we did not measure renin at the same time to determine if the serum level of renin in diabetes increased or not. Renin is well known to be a key enzyme in the cleavage of angiotensinogen to angiotensin $\mathrm{I}$, and this reaction is a rate limiting step to generate angiotensin II in the reninangiotensin system (RAS). Previous reports showed that the concentration of renin in diabetes does not increase, ${ }^{21}$ although RAS has been implicated in the pathogenesis of DR. $^{3-5}{ }^{15} 22-25$ The fact that renin does not increase in diabetes seems to be a discrepancy, but RAS is activated in diabetes. Our study, as other previous reports showed, ${ }^{3-51326}$ might indicate the involvement of increased prorenin in the development of DR. In addition, as mentioned previously, the plasma concentration of prorenin precedes the occurrence of diabetic nephropathy by several years. ${ }^{78}$ Increasing prorenin in diabetes may trigger microangiopathy and promote the development of diabetic microangiopathy through the activation of RAS. 
Recently, prorenin was reported to have enzymatic activity that generated angiotensin I and activated the RAS thorough the generation of angiotensin II. ${ }^{26-28}$ Prorenin is composed of two components, the profragment of prorenin and mature renin. Suzuki et al demonstrated that prorenin has a key region in its profragment for non-proteolytic activation with protein interaction. ${ }^{26}$ Furthermore, Nguyen et al, who investigated the renin/prorenin receptor, showed that the prorenin binding this receptor activated the conversion of angiotensinogen to angiotensin I. High levels of this receptor mRNA were detected in the heart, brain, and placenta and lower levels in the kidney and liver. ${ }^{27}$ Recently, Ichihara et al proved the non-proteolytic activation of prorenin and the presence of renin/prorenin receptor in the kidney using streptozotocin induced diabetic rats. They reported that the interference of prorenin with peptide which inhibits an interaction of prorenin with renin/prorenin receptor, inhibited the local generation of angiotensin II and improved diabetic nephropathy in streptozotocin induced diabetic rats. ${ }^{18}$ Angiotensin I generated with non-proteolytic activation of prorenin is transformed to angiotensin II by soluble or endothelium specific angiotensin converting enzyme. Angiotensin II exhibits pathological effects in the retina in diabetes through binding angiotensin II type l receptor, which is thought to be the most important receptor of all subtypes to exhibit the physiological and pathological effects. Angiotensin II is associated with overexpression of some angiogenic factors-that is, vascular endothelial growth factor (VEGF), ${ }^{29-33}$ and angiopoietin $2 .{ }^{34}$ VEGF and angiopoietin 2 have a crucial role in the development of retinal neovascularisation, ${ }^{35-38}$ a main feature of PDR. Taken together, it is possible that a high concentration of prorenin in patients with diabetes activates the local RAS in the eyes through its binding renin/prorenin receptor and promotes the pathogenesis of DR through the generation of angiotensin II.

In this study, we evaluated the serum levels of prorenin in patients with type 2 diabetes with a newly developed method, the AAD-PR assay. The serum levels of prorenin in patients with PDR were markedly high. High levels of prorenin in diabetes increase with increasingly severe retinopathy. We showed that a high concentration of circulating prorenin may be involved in the pathogenesis of DR. Further prospective study is needed to investigate the relation between modulation of the serum levels of prorenin and the severity of DR, and in turn, whether patients without DR with a higher level of prorenin will develop retinopathy.

\section{Authors' affiliations}

H Yokota, F Mori, K Kai, T Nagaoka, N Izumi, A Takahashi, T Hikichi, A Yoshida, Department of Ophthalmology, Asahikawa Medical College, Asahikawa, Japan

F Suzuki, Faculty of Applied Biological Science, Gifu University, Gifu, Japan

Y Ishida, Preveqol Co, Tokyo, Japan

The authors have no proprietary interest in any aspect of this report.

\section{REFERENCES}

1 The Diabetes Control and Complications Trial Research Group. The effect of intensive treatment of diabetes on the development and progression of longterm complications in insulin-dependent diabetes mellitus. N Engl J Med 1993;329:977-86.

2 Mattock MB, Cronin N, Cavallo-Perin P, et al. Plasma lipids and urinary albumin excretion rate in Type 1 diabetes mellitus: the EURODIAB IDDM Complications Study. Diabet Med 2001;18:59-67.

3 Luetscher JA, Kraemer FB, Wilson DM, et al. Increased plasma inactive renin in diabetes mellitus. A marker of microvascular complications. N Engl J Med 1985;312:1412-17

4 Franken AA, Derkx FH, Schalekamp MA, et al. Association of high plasma prorenin with diabetic retinopathy. J Hypertens Suppl 1988;6:S461-3.

5 Franken AA, Derkx FH, Blankestijn PJ, et al. Plasma prorenin as an early marker of microvascular disease in patients with diabetes mellitus. Diabete Metab 1992;18:137-43.
6 Allen TJ, Cooper ME, Gilbert RE, et al. Serum total renin is increased before microalbuminuria in diabetes. Kidney Int 1996;50:902-7.

7 Deinum J, Ronn B, Mathiesen E, et al. Increase in serum prorenin precedes onset of microalbuminuria in patients with insulin-dependent diabetes mellitus. Diabetologia 1999:42:1006-10.

8 Chiarelli F, Pomilio M, De Luca FA, et al. Plasma prorenin levels may predict persistent microalbuminuria in children with diabetes. Pediatr Nephrol 2001;16:116-20.

9 Kordonouri O, Wladimirowa A, Danne T. High total serum renin concentrations are associated with the development of background retinopathy in adolescents with type 1 diabetes. Diabetes Care 2000;23:1025-6.

10 Sealey JE. Plasma renin activity and plasma prorenin assays. Clin Chem 1991;37:1811-19.

11 Cooper RM, Murray GE, Osmond DH. Trypsin-induced activation of renin precursor in plasma of normal and anephric man. Circ Res 1977;40:1171-9.

12 Derkx FH, de Bruin RJ, van Gool JM, et al. Clinical validation of renin monoclonal antibody-based sandwich assays of renin and prorenin, and use of renin inhibitor to enhance prorenin immunoreactivity. Clin Chem 1996;42:1051-63.

13 Suzuki F, Hatano Y, Nakagawa T, et al. Non-proteolytic activation of human prorenin by anti-prorenin prosegment (pf\#1: 1P-15P) antiserum. Biosci Biotechnol Biochem 1999;63:550-4.

14 Kawazu S, Minagawa S, Yazawa M, et al. Sex difference and possible relationship to microvascular complications of serum prorenin levels in type 2 diabetic patients, measured by a novel antibody-activating direct enzyme kinetic assay. Journal of Diabetes and lts Complications 2004;18:275-81.

15 Makimattila S, Summanen P, Matinlauri I, et al. Serum total renin, an independent marker of the activity and severity of retinopathy in patients with IDDM. Br J Ophthalmol 1998;82:939-44.

16 Sealey JE, Glorioso N, Itskovitz J, et al. Prorenin as a reproductive hormone. New form of the renin system. Am J Med 1986;81:1041-6.

17 Ubeda M, Hernandez I, Fenoy F, et al. Vascular and adrenal reninlike activity in chronically diabetic rats. Hypertension 1988;11:339-43.

18 Ichihara A, Hayashi M, Kaneshiro Y, et al. Inhibition of diabetic nephropathy by a decoy peptide corresponding to the "handle" region for nonproteolytic activation of prorenin. J Clin Invest 2004; 114:1128-35.

19 Chaturvedi N, Sjoelie AK, Porta M, et al. Markers of insulin resistance are strong risk factors for retinopathy incidence in type 1 diabetes. Diabetes Care 2001; $24: 284-9$.

20 Kohner EM, Aldington SJ, Stratton IM, et al. United Kingdom Prospective Diabetes Study, 30: diabetic retinopathy at diagnosis of non-insulindependent diabetes mellitus and associated risk factors. Arch Ophthalmol 1998; 116:297-303.

21 Derkx FH, Schalekamp MA. Human prorenin: pathophysiology and clinical implications. Clin Exp Hypertens A 1988;10:1213-25.

22 Drury PL, Bodansky HJ, Oddie CJ, et al. Increased plasma renin activity in type 1 diabetes with microvascular disease. Clin Endocrinol 1982;16:453-61.

23 Moravski CJ, Skinner SL, Stubbs AJ, et al. The renin-angiotensin system influences ocular endothelial cell proliferation in diabetes: transgenic and interventional studies. Am J Pathol 2003; 162:151-60.

24 Funatsu $\mathrm{H}$, Yamashita $\mathrm{H}$. Pathogenesis of diabetic retinopathy and the reninangiotensin system. Ophthalmic Physiol Opt 2003;23:495-501.

25 Siolie AK, Chaturvedi N. The retinal renin-angiotensin system: implications for therapy in diabetic retinopathy. J Hum Hypertens 2002;16(Suppl 3):S42-6.

26 Suzuki F, Hayakawa M, Nakagawa T, et al. Human prorenin has "gate and handle" regions for its non-proteolytic activation. J Biol Chem 2003;278:22217-22.

27 Nguyen G, Delarue F, Burckle C, et al. Pivotal role of the renin/prorenin receptor in angiotensin II production and cellular responses to renin. J Clin Invest 2002;109:1417-27.

28 Prescott G, Silversides DW, Reudelhuber TL. Tissue activity of circulating prorenin. Am J Hypertens 2002;15:280-5.

29 Wilkinson-Berka JL, Kelly DJ, Gilbert RE. The interaction between the reninangiotensin system and vascular endothelial growth factor in the pathogenesis of retinal neovascularization in diabetes. J Vasc Res 2001;38:527-35.

30 Sarlos S, Rizkalla B, Moravski CJ, et al. Retinal angiogenesis is mediated by an interaction between the angiotensin type 2 receptor, VEGF, and angiopoietin. Am J Pathol 2003; 163:879-87.

31 Otani A, Takagi H, Suzuma K, et al. Angiotensin II potentiates vascular endothelial growth factor-induced angiogenic activity in retinal microcapillary endothelial cells. Circ Res 1998;82:619-28.

32 Moravski CJ, Kelly DJ, Cooper ME, et al. Retinal neovascularization is prevented by blockade of the renin-angiotensin system. Hypertension 2000;36: 1099-104.

33 Williams B. A potential role for angiotensin II-induced vascular endothelial growth factor expression in the pathogenesis of diabetic nephropathy? Miner Electrolyte Metab 1998;24:400-5.

34 Otani A, Takagi $\mathrm{H}, \mathrm{Oh} \mathrm{H}$, et al. Angiotensin II induces expression of the Tie2 receptor ligand, angiopoietin-2, in bovine retinal endothelial cells. Diabetes 2001;50:867-75.

35 Oh H, Takagi H, Suzuma K, et al. Hypoxia and vascular endothelial growth factor selectively up-regulate angiopoietin-2 in bovine microvascular endothelial cells. J Biol Chem 1999;274:15732-9.

36 Takagi H, Koyama S, Seike H, et al. Potential role of the angiopoietin/tie2 system in ischemia-induced retinal neovascularization. Invest Ophthalmol Vis Sci 2003:44:393-402.

37 Umeda N, Ozaki H, Hayashi $\mathrm{H}$, et al. Colocalization of Tie2, angiopoietin 2 and vascular endothelial growth factor in fibrovascular membrane from patients with retinopathy of prematurity. Ophthalmic Res 2003;35:217-23.

38 Oshima Y, Deering T, Oshima S, et al. Angiopoietin-2 enhances retinal vessel sensitivity to vascular endothelial growth factor. J Cell Physiol 2004;199:412-17. 